[Chem. Pharm. Bull.

35(12)4946-4952(1987) $]$

\title{
Effect of Sonication on the Dispersion State of Lipopolysaccharide and Its Pyrogenicity in Rabbits
}

\author{
Tetsuo Komuro, ${ }^{*}$ Toshimi Murai and Hironoshin Kawasaki \\ National Institute of Hygienic Sciences, Osaka, Hoenzaka 1-1-43, \\ Higashi-ku, Osaka 540, Japan
}

(Received June 18, 1987)

\begin{abstract}
The effect of sonication on the particle size of lipopolysaccharide (LPS) in aqueous media was studied, in order to examine the relation of particle size to pyrogenicity in rabbits, by the sucrose density gradient ultracentrifugation technique. LPS extracted from $E$. coli UKT-B according to the phenol/water method showed a polydispersed profile on the gradients, but after sonication for 3 min it formed a single peak in the lower density regions. From the results of electron micrographic observations, partial specific volume, viscosity and turbidity measurements, and density gradient data, it was revealed that sonication produced a decrease in the particle size of LPS. A more marked pyrogenicity in rabbits was observed in the LPS fractions in the lower density regions than in the higher ones, or in the fractions having smaller-sized particles of LPS than in the fractions having larger particles.
\end{abstract}

Keywords-lipopolysaccharide; LPS; LAL test; sonication; pyrogenicity

\section{Introduction}

Pyrogenicity is well known to be one of the fundamental biological activities elicited by lipopolysaccharide (LPS) ${ }^{1,2)}$ of gram-negative bacteria, and the biological activities of LPS, including pyrogenicity, are suggested to be greatly affected by the particle size, or state of dispersion, ${ }^{3-11)}$ since LPS extracted by the phenol/water method is usually considerably aggregated in aqueous media. ${ }^{12,13)}$ During our studies on the pyrogenicity of LPS preparations, we found that sonication for 2 or $3 \mathrm{~min}$ significantly enhanced their pyrogenicity in rabbits; a part of these results was reported at the 28 th Symposium on Endotoxins in Japan. ${ }^{14)}$ This result suggests that sonication might disperse the aggregates of LPS into smaller and more uniform particles with an optimum size for manifesting biological activities in aqueous media, resulting in an enhancement of pyrogenicity. This phenomenon is noteworthy because the pyrogenicity of LPS was affected significantly by this physical procedure, which is fundamentally different from the action of surfactants such as sodium deoxycholate, which dissociate the aggregates of LPS mainly by chemical forces into subunits and simultaneously reduce the pyrogenicity. ${ }^{6,9)}$ In investigating what size of LPS particle is required for optimum biological activities, sonication seems to be a useful tool. This study was, therefore, undertaken to investigate in detail the dispersion state of LPS brought about by sonication in aqueous media by using the sucrose density gradient ultracentrifugation (SDGU) technique, in order to examine the relation of particle size of LPS to pyrogenicity and Limulus amoebocyte lysate (LAL) gelation activity.

\section{Experimental}

LPS _ LPS was extracted from E. coli UKT-B according to the hot phenol/water method reported by Westphal et al. ${ }^{15)}$ Crude LPS was purified initially with $50 \%$ ethyl alcohol, twice, to yield semi-purified LPS and 
further purified by ultracentrifugation in a Hitachi $55 \mathrm{P}-2$ centrifuge $(100000 \times \mathrm{g}, 2 \mathrm{~h})$ until the ribonucleic acid content of the LPS preparation became negligible.

Sonication of LPS - LPS was suspended in sterilized, distilled water at the concentration of $1 \mathrm{mg} / \mathrm{ml}$ and sonicated for a definite period in an ice-bath using a Nihon Seiki ultrasonic generator, model US-3 $(30 \mathrm{~W} / 28 \mathrm{kHz})$.

SDGU Analysis of LPS - Linear density gradients having $4.5 \mathrm{ml}$ of 5 to 40 or 2 to $16 \%(\mathrm{w} / \mathrm{w})$ sucrose were prepared with a Hitachi gradient maker, model DGK-U. LPS was suspended in sterilized, distilled water at the concentration of $1 \mathrm{mg} / \mathrm{ml}$; when necessary, the suspension was sonicated for a definite period, then $200 \mu 1$ was layered onto the gradients. SDGU was performed at $100000 \times g$ for 90 or $240 \mathrm{~min}$ at $4^{\circ} \mathrm{C}$ using a Hitachi swinging rotor, model RPS-40. Fractions of $200 \mu \mathrm{l}$ were collected from the bottom of the tube through a needle inserted from above to yield 23 fractions, which were analyzed for LPS by the carbocyanine dye assay procedures described below. Sucrose was confirmed not to disturb this assay. Sucrose density of each fraction was measured with an Abbe refractometer (Atago Co., Ltd.).

Electron Micrographical Analysis of LPS _ LPS samples for electron micrography were negatively stained with $3 \%$ phosphotungstic acid, $\mathrm{pH} 7.3$, on carbon films and rendered hydrophilic by a glow discharge in air. Micrographs were recorded at an instrument magnification of 23000 (JEM-100U electron microscope, Nihon Denshi Co., Ltd).

Partial Specific Volume, Viscosity and Turbidity Measurements of LPS_Partial specific volume of LPS was measured by using a density column consisting of bromobenzene and xylene according to the usual method. ${ }^{16)}$ Relative viscosity of LPS was measured in the presence of $0.05 \mathrm{M} \mathrm{NaCl}$ at $20^{\circ} \mathrm{C}$ in an Ubbelohde-type viscometer. Turbidity experiments were performed by measuring the absorbance of LPS at $520 \mathrm{~nm}$ spectrophotometrically.

Chemical Analysis of LPS__ LPS was determined colorimetrically by means of the carbocyanine dye reaction according to the method of Zey and Jackson. ${ }^{17)}$ Carbocyanine dye, 1-ethyl-2-[3-(1-ethylnaphtho[1,2d]thiazoline-2ylidene)-2-methylpropenyl]naphtho[1,2d]thiazolium bromide, was obtained from Eastman Kodak Co.

Pyrogen Test of LPS in Rabbits - The pyrogenicity of LPS samples was tested in Japanese white male rabbits weighing about $3 \mathrm{~kg}$ in an air-conditioned room (temp. $24 \pm 1{ }^{\circ} \mathrm{C}$, relative humidity $55 \pm 5 \%$ ). Not less than three animals were used in each experiment. LPS was fractionated by the SDGU technique described above and the fractions were assayed for LPS according to the carbocyanine dye method. Fractions were diluted with sterile saline to give a definite concentration of LPS. Rabbits were injected with $0.01 \mu \mathrm{g}$ of LPS per kg of body weight intravenously and changes in rectal temperature were recorded automatically for $4 \mathrm{~h}$. Maximum rectal temperature increase $\left(\Delta T_{\max }\right)$ and fever index for $4 \mathrm{~h}$ (FI-4), representing the area under the fever curve for $4 \mathrm{~h}$ were determined as described previously. ${ }^{14)}$

LAL Test of LPS _ LAL tests were performed to detect LPS in test solutions semi-quantitatively. ${ }^{18)}$ The capacity of the endotoxins to cause gelation of the lysate from amoebocytes of Limulus polyphemus was estimated with the use of "Pre gel" (Seikagaku Kogyo Co., Ltd.) according to the usual method. Test solutions were made by serial tenfold dilution of samples containing LPS. When necessary, further twofold dilutions were made and endpoint dilution was recorded. The test results were scored as ++ (firm gel), + (loose gel) and - (slight flocculation).

Reagents - Standard ribonucleic acid (RNA) with known sedimentation coefficients $(23,16$ and $5+6 \mathrm{~S})$ were purchased from Miles Laboratories Inc. All other reagents used in these experiments were of analytical grade and were purchased from commercial sources.

\section{Results}

\section{Pyrogenicity and LAL Gelation Activity of LPS Fractionated by SDGU}

Non-sonicated LPS was fractionated by the SDGU technique and the LPS content of each fraction was determined colorimetrically by application of the carbocyanine dye reaction. The results obtained are shown at the bottom of Fig. 1. Based on the amount of LPS determined in this way, a definite amount of LPS in each fraction was subjected to pyrogen test in rabbits and to the LAL test. Maximum rectal temperature increase and intensity of febrile response for $4 \mathrm{~h}$ were estimated and are represented in the middle of Fig. 1 as $\Delta T_{\max }$ and FI-4, respectively. Stronger febrile responses in rabbits were observed in the LPS fractions in the lower density regions than in the higher density ones. This result agreed well with that of the LAL test as shown at the top of Fig. 1.

\section{Dispersion Profile of LPS by SDGU}

To clarify the effect of sonication on the particle size of LPS, the SDGU profile of LPS was investigated. LPS suspensions at the concentration of $1 \mathrm{mg} / \mathrm{ml}$ were sonicated for $1,3,10$ or $30 \mathrm{~min}$ and the results obtained are shown in Fig. 2. In the case of non-sonicated LPS, LPS 


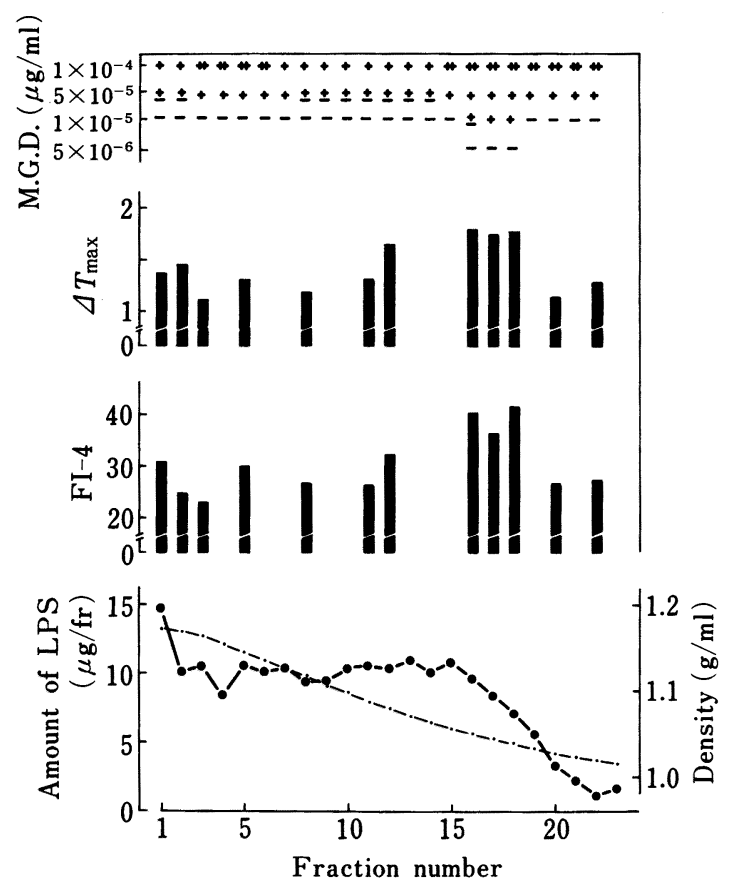

Fig. 1. Sucrose Density Gradient Ultracentrifugation Profile of LPS and Distribution Patterns of Pyrogenicity in Rabbits and LAL Gelation Activity

M.G.D., minimum gelation dose; FI-4, fever index for $4 \mathrm{~h}$ (representing area under the fever curve); $\Delta T_{\max }$, maximum increase in rectal temperature for $4 \mathrm{~h}$. The linear density gradient was 5 to $40 \%(w / w)$ of sucrose. Centrifugation was done at $100000 \times g$ for $90 \mathrm{~min}$ at $4^{\circ} \mathrm{C}$. The LPS dose in pyrogen tests was $0.01 \mu \mathrm{g} / \mathrm{kg}$ of body weight. Each bar indicates a mean of not less than three experiments.

was found to be distributed almost evenly throughout the gradient, suggesting the presence of various sizes of LPS particles in aqueous media. However, the SDGU profile of sonicated LPS was different from that of the non-sonicated LPS, that is, a peak appeared in the profile and the peak shifted gradually toward the lower-density regions with increased period of sonication. This suggests that larger particles of LPS became smaller under the influence of sonication. However, sonication for more than $10 \mathrm{~min}$ no longer caused a positional change of the peak except for a small decrease of the peak area and a corresponding increase in the amount near the top of the gradient.

\section{Particle Size of LPS Sonicated for 3 min}

The particle size of sonicated LPS $(3 \mathrm{~min})$ manifesting more potent pyrogenicity and LAL gelation activity as shown in Fig. 1, was estimated by using standard RNA with known sedimentation coefficients (S) of 23, 16 and 5+6S as markers. The result is shown in Fig. 3, suggests that LPS particles sonicated for $3 \mathrm{~min}$ have a definite size corresponding to standard RNA with a sedimentation coefficient of between 16 and $23 \mathrm{~S}$.

\section{Electron Micrography of LPS}

The particle size of LPS was directly observed by electron micrography. The sizes of about 2000 particles of sonicated LPS were measured on the micrographs and compared with those of non-sonicated LPS as a control. The results obtained are presented in Table I, and some examples are shown in Fig. 4. It was revealed that non-sonicated LPS was variable in length, but that after 2 min of sonication, LPS particles with a length of more than $320 \mathrm{~nm}$ 


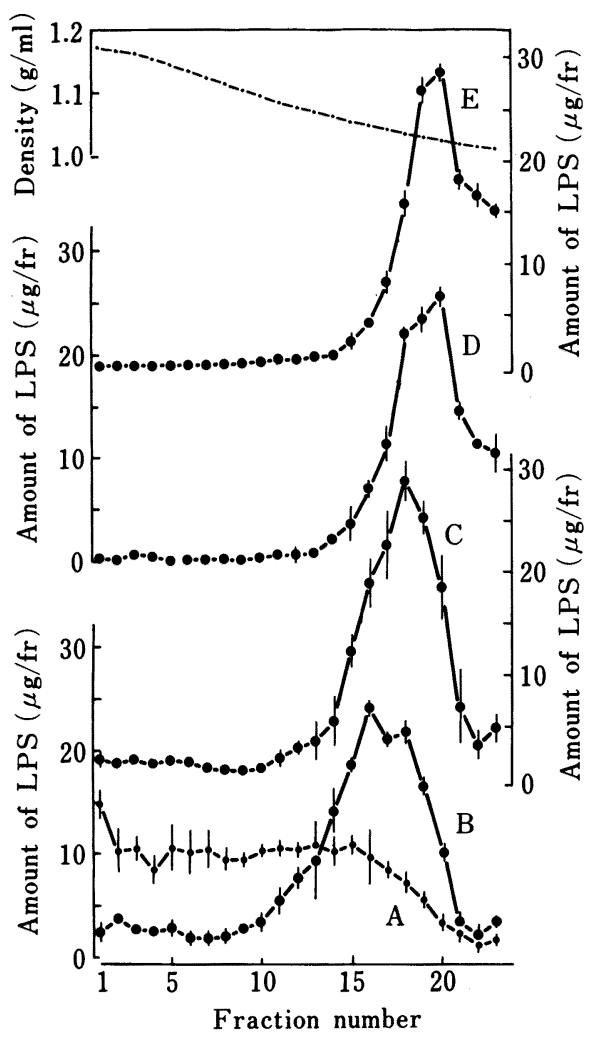

Fig. 2. Sucrose Density Gradient Profiles of Sonicated LPS

Sonication time: A, 0; B, 1; C, 3; D, 10; E, $30 \mathrm{~min}$ The linear density gradient was 5 to $40 \%(\mathrm{w} / \mathrm{w})$ of sucrose. Centrifugation was done at $100000 \times g$ for $90 \mathrm{~min}$ at $4^{\circ} \mathrm{C}$. Each point with bar indicates a mean \pm S.D. of three experiments.

-- , density; - - , amount of LPS.

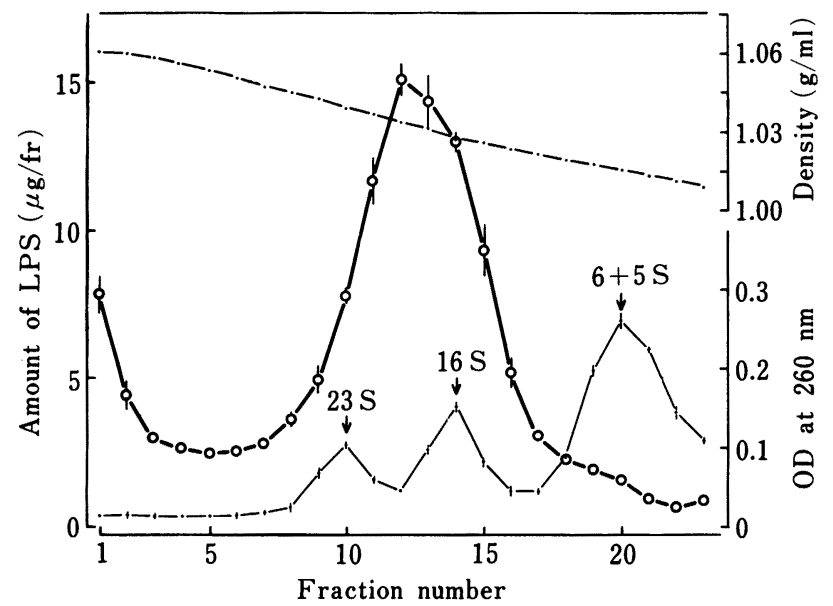

Fig. 3. Comparison of the Density Gradient Profile of Sonicated (3 min) LPS with That of Standard RNA

The linear gradient was 2 to $16 \%(\mathrm{w} / \mathrm{w})$ of sucrose in water for LPS and in $15^{-1} \mathrm{M}$ phosphate buffer solution, $\mathrm{pH} 7.0$, for RNA. Centrifugation was done at $100000 \times \boldsymbol{g}$ for 240 min at $4{ }^{\circ} \mathrm{C}$. Each point with bar indicates a mean \pm S.D. of three experiments.

$-\mathrm{O}-$, amount of LPS; - - , OD at $260 \mathrm{~nm}$.

disappeared completely, while after $30 \mathrm{~min}$ of sonication, most particles fell within the range of less than $20 \mathrm{~nm}$ in length. These results demonstrate that the particle size of LPS was decreased by sonication. 
TABLE I. Effect of Sonication on Particle Length of LPS

\begin{tabular}{cccc}
\hline \hline \multirow{2}{*}{$\begin{array}{c}\text { Range } \\
(\mathrm{nm})\end{array}$} & \multicolumn{3}{c}{ Percentage of LPS particles sonicated for } \\
\cline { 2 - 4 } & 0 min & 2 min & 30 min \\
\hline $0-10$ & 2.4 & 3.1 & 21.6 \\
$10-20$ & 13.5 & 19.3 & 61.2 \\
$20-40$ & 12.3 & 19.9 & 12.9 \\
$40-80$ & 18.1 & 30.4 & 4.0 \\
$80-160$ & 24.0 & 25.9 & 0.0 \\
$160-320$ & 13.8 & 1.5 & 0.0 \\
$320-$ & 15.9 & 0.0 & 0.0 \\
\hline
\end{tabular}

Based on micrographs of LPS sonicated for 2 or $3 \mathrm{~min}$, the lengths of 2000 LPS particles were measured and the distribution pattern (percentage) was calculated.

(A)

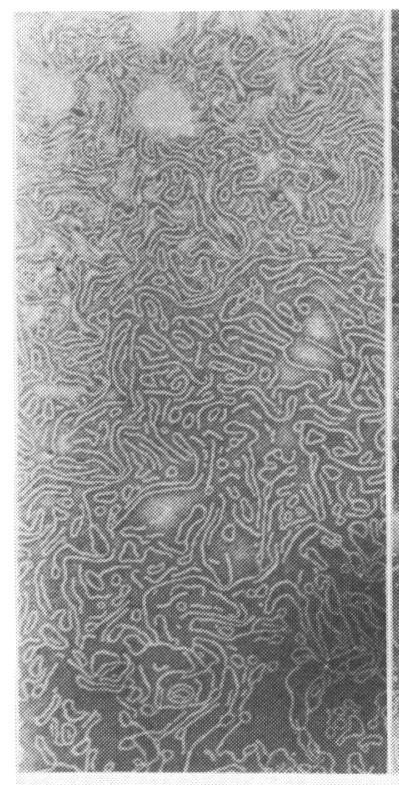

(B)

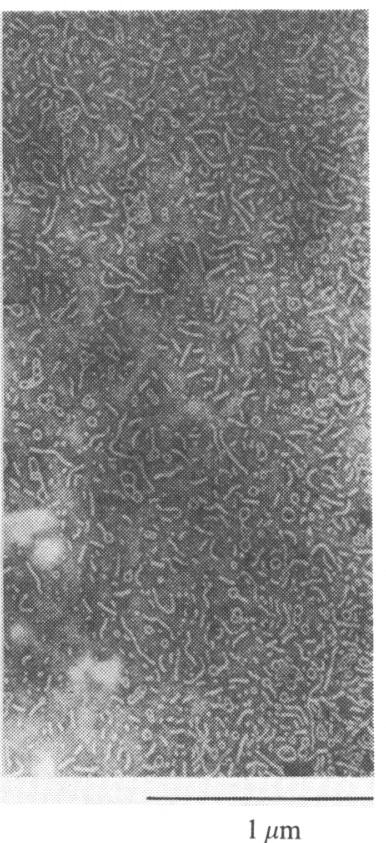

Fig. 4. Electron Micrographs of Negatively Stained Preparations of Phenol/WaterExtracted LPS from E. coli UKT-B

(A) Non-sonicated LPS, (B) sonicated (2 min) LPS.

\section{Partial Specific Volume, Viscosity and Turbidity of LPS}

To clarify the effect of sonication on the particle size of LPS, some physicochemical properties were investigated. First, the partial specific volume of LPS was measured by using a density column consisting of bromobenzene and xylene. The value of the partial specific volume of non-sonicated LPS was approximately $0.717 \mathrm{ml} / \mathrm{g}$, and did not change after sonication for $1,2,3,5,10$ or $30 \mathrm{~min}$.

Next, viscosity and turbidity changes of LPS induced by sonication were investigated. The results obtained are shown in Fig. 5. Sonication for a few minutes caused a remarkable fall in the viscosity of LPS, but the viscosity did not change on further sonication. A similar result was observed in the turbidity change of LPS as shown in Fig. 5. These results indicate 


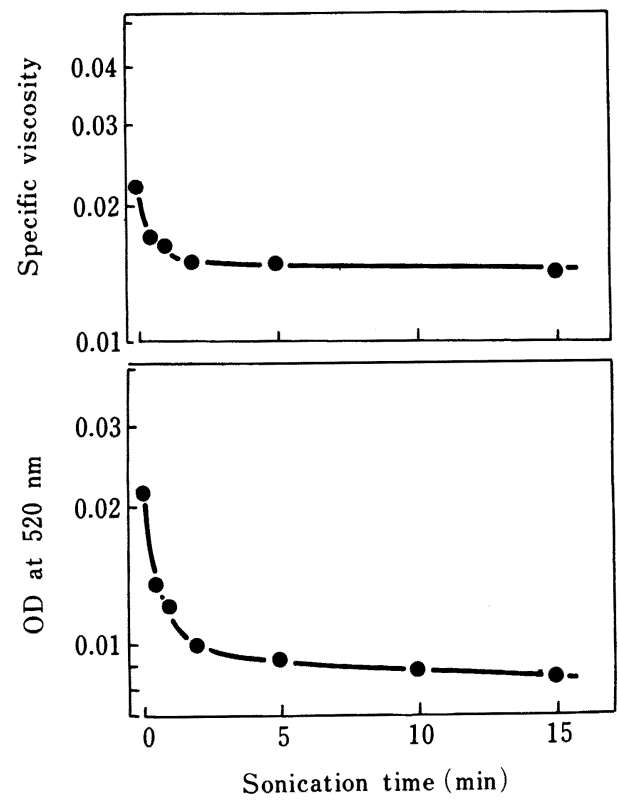

Fig. 5. Changes in the Viscosity and Turbidity of LPS Induced by Sonication

that the particle size of LPS was decreased by sonication.

\section{Discussion}

LPS of gram-negative bacteria is usually composed of two contrasting parts, a hydrophilic part and a hydrophobic part, and is therefore amphiphilic. ${ }^{19,20)}$ In spite of its favorable characteristics, it is only slightly soluble in water as well as in organic solvents. As pointed out previously, ${ }^{21,22)}$ this property has made the study of LPS difficult. In measuring not only physicochemical constants of LPS but also its biological activities, careful consideration must be given to the dispersion state of LPS in aqueous media. Our data indicated that the dispersion state of LPS in aqueous media was not always uniform in terms of the aggregate size, and our attention was, therefore, focused on the effect of sonication, which is most frequently used as a means to disperse or dissolve slightly soluble materials uniformly in aqueous media. ${ }^{23.24)}$

Sonication caused a stepwise shift in the sedimentation profile of LPS on the sucrose gradient, which may not always directly reflect a decrease in the particle size of LPS. The change in sedimentation behavior observed in the present experiment is, however, considered to reflect a decrease in the particle size of LPS from the present results, i.e., (1) that electron micrographic observations directly showed the decrease in the particle size of LPS by sonication, (2) that the partial specific volume of sonicated LPS was identical to that of nonsonicated LPS and (3) that the viscosity and turbidity of LPS were markedly decreased by sonication, and in addition, from other evidence that disaggregation of LPS lowers the sedimentation coefficient of LPS. ${ }^{25}{ }^{26)}$ Based on these observations, studies of the dispersion state of LPS in aqueous media were carried out in order to examine the relation of particle size to biological activities of LPS. As shown in Fig. 2, sonication-induced disaggregation of LPS was demonstrated by SDGU. In other words, appropriate sonication made it possible for us to investigate LPS of limited size that retained the intact properties. Using this method, we found a potent pyrogenicity and LAL gelation activity of LPS in the lower density regions near 1.04 density on the sucrose gradient under the present experimental conditions, as shown 
in Fig. 2, though this size is not the minimum that can be produced by the sonication (Fig. 2). These results strongly suggest that there might be a definite optimum particle size of LPS required for the manifestation of pyrogenicity in rabbits and for LAL gelation activity.

There are reports describing a reduction in pyrogenicity of LPS by a simple dissociation of LPS particles into subunits using surfactants such as sodium deoxycholate. ${ }^{6,9}$ However, in those experiments LPS has not always remained in an intact state. That is, considering that LPS was treated with surfactants to reduce its particle size, there remains a possibility that active sites of LPS might be masked by forming a complex with surfactants, ${ }^{2,11)}$ resulting in a reduction of the pyrogenicity. To resolve this problem, it will be necessary to dissociate large particles of LPS into smaller ones without surfactants, or without undesirable modifications, and to isolate them for pyrogen test in rabbits.

The present experimental results showed that sonication decreased the particle size of LPS and that the most potent febrile response in rabbits was produced by smaller-particle-sized LPS in the lower density regions near 1.04 density on the sucrose gradient under the present experimental conditions. Sonication will be useful for studying the biological activities of LPS because it produces uniform-sized particles of LPS which show optimum activity and because smaller particles of LPS produced by sonication still retain the intact properties, probably without any modification except particle size.

\section{References}

1) R. J. Elin and S. M. Wolff, Annu. Rev. Med., 27, 127 (1976).

2) A. Nowotony, Bacterial. Rev., 33, 79 (1969).

3) B. S. Roberson and W. J. Cromartie, J. Bacteriol., 84, 882 (1962).

4) E. Ribi, W. T. Haskin, K. C. Milner, R. T. Anacker, D. B. Ritter, G. Goode, R. J. Trapani and M. Landy, J. Bacteriol., 84, 803 (1962).

5) K. C. Milner, K. Fukushi, W. T. Haskin, M. Landy, B. Malmgren and E. Ribi, Bacteriol. Rev., 27, 352 (1963).

6) E. Ribi, R. L. Anacker, R. Brown, W. T. Haskin, B. Malmgren, K. C. Milner and J. A. Rudbach, J. Bacteriol., 92, 1493 (1966).

7) F. C. McIntire, H. W. Sievert, G. H. Barlow, R. A. Finley and A. Y. Lee, Biochemistry, 6, 2363 (1967).

8) D. F. Tarmina, K. C. Milner, E. Ribi and J. A. Rudbach, J. Bacteriol., 96, 1611 (1968).

9) D. F. Tarmina, K. C. Milner, E. Ribi and J. A. Rudbach, J. Immunol., 100, 444 (1968).

10) A. L. Jackson, J. Bacteriol., 97, 13 (1969).

11) F. C. McIntire, G. H. Barlow, H. W. Sievert, R. A. Finley and A. L. Yoo, Biochemistry, 8, 4063 (1980).

12) J. W. Shands and P. W. Chun, J. Biol. Chem., 255, 1221 (1980).

13) O. Westphal and K. Jann, Methods Carbohydrate Chem., 5, 83 (1965).

14) S. Kanoh, Y. Ogawa, T. Komuro and H. Kawasaki, Jpn. J. Med. Sci. Biol., 35, 112 (1982).

15) O. Westphal, O. Lüderitz and F. Bister, Z. Naturforshg., 7 b, 148 (1952).

16) Nihon Seibutsu Butsuri Gakkai (ed.), "Zoku Seibutsu Butsuri Gaku Kōza, Butsuri-teki Sokutei-hou II," Yoshioka Shoten, Tokyo, 1968, p. 209.

17) P. Zey and S. Jackson, Appl. Microbiol., 26, 129 (1973).

18) J. Levin, "Biochemical Applications of the Horseshoe Crab (Limulidate)," ed. by E. Cohen, Alan R. Liss, Inc., New York, 1978, p. 131.

19) J. M. Shands, J. A. Graham and K. Nath, J. Mol. Biol., 25, 15 (1967).

20) O. Westphal, Int. Archs. Allergy Appl. Immunol., 49, 1 (1975).

21) A. Nowotony, Ann. N. Y. Acad. Sci., 133, 586 (1966).

22) H. Beer, A. I. Braude and C. C. Brinton, Ann. N. Y. Acad. Sci., 133, 450 (1966).

23) L. Saunders, J. Perrin and D. Gammack, J. Pharm. Pharmacol., 14, 567 (1962).

24) D. Attwood and L. Saunders, Biochem. Biophys. Acta, 98, 344 (1965).

25) C. Galanos and O. Lüderitz, Eur. J. Biochem., 54, 603 (1975).

26) K. A. Johnson, P. A. Ward, S. Goralnick and M. J. Osborn, Am. J. Physiol., 88, 559 (1977). 